\title{
Aprendizaje colaborativo y lectoescritura: un análisis con estudiantes de educación general básica
}

\section{Collaborative learning and literacy: an analysis with basic general education students}

\author{
Luz María Arias-Jaramillo \\ luz.arias@psg.ucacue.edu.ec \\ Universidad Católica de Cuenca, Azogues \\ Ecuador \\ https://orcid.org/0000-0002-6075-4897 \\ Darwin Gabriel García-Herrera \\ dggarciah@ucacue.edu.ec \\ Universidad Católica de Cuenca, Azogues \\ Ecuador \\ https://orcid.org/0000-0001-6813-8100 \\ Ana Zulema Castro-Salazar \\ azcastros@ucacue.edu.ec \\ Universidad Católica de Cuenca, Azogues \\ Ecuador \\ https://orcid.org/0000-0002-3837-314X \\ Juan Carlos Erazo-Álvarez \\ jcerazo@ucacue.edu.ec \\ Universidad Católica de Cuenca, Cuenca \\ Ecuador \\ https://orcid.org/0000-0001-6480-2270
}

Recepción: 09 abril 2020

Revisado: 15 de mayo 2020

Aprobación: 15 junio 2020

Publicación: 1 de julio 2020 


\title{
RESUMEN
}

El objetivo fue analizar el uso del aprendizaje colaborativo para desarrollar la lectoescritura en Educación General Básica del Circuito 06_07_08_09, perteneciente al Distrito 07D02, Machala, de carácter descriptivo correlacional no experimental. Los niveles de confiabilidad permitieron demostrar estadísticamente la relación existente entre el aprendizaje colaborativo y lectoescritura, la participación docente es importante brindando la oportunidad de mejorar el proceso de enseñanza-aprendizaje, la propuesta planteada resalta la incorporación de metodologías activas, a través del aprendizaje colaborativo.

Descriptores: Autoaprendizaje; experimento educacional; hábito de lectura; promoción de la lectura. (Palabras tomadas del Tesauro UNESCO).

\begin{abstract}
The objective was to analyze the use of collaborative learning to develop literacy in Basic General Education of Circuit 06_07_08_09, belonging to District 07D02, Machala, of a non-experimental correlational descriptive nature. The levels of reliability allowed to statistically demonstrate the relationship between collaborative learning and literacy, teacher participation is important providing the opportunity to improve the teachinglearning process, the proposed proposal highlights the incorporation of active methodologies, through collaborative learning.
\end{abstract}

Descriptors: Self instruction; educational experiments; reading habit; reading promotion. (Words taken from the UNESCO Thesaurus). 


\section{INTRODUCCIÓN}

La utilización de metodologías activas a través del trabajo colaborativo, permite alcanzar notablemente el aprendizaje-significativo de los estudiantes, es decir, logrando que los niños sean los protagonistas de sus propios conocimientos durante todo el proceso educativo. (Ballestas Camacho, 2015) en un estudio realizado en Colombia, menciona que el aprendizaje de la lectoescritura es trascendentales en el desarrollo de un niño, enseñar a leer, a escribir constituye el retos más importantes de todas las escuelas, siendo una habilidad vinculada al aprendizajes, cabe señalar, que se considera un eslabón fundamental durante todo el proceso educativo, resultando ser la piedra angular en el desarrollo académico del estudiantes.

(Cano de la Cruz, Aguiar Monar, \& Mendoza Román, 2017) llevaron a cabo el estudio en Ecuador, sobre metodología activas, obteniendo como resultados insuficiente conocimiento por parte del grupo docente. Encontrándose con este impedimento que conlleva al fracaso, retaso en el avance académico de los niños, se debe aclarar que mientras exista el mínimo interés y compromiso por parte del docente, muy distante estará la enseñanza de un aprendizaje significativo formando entes poco participativos en las diferentes clases, convirtiéndolos seres conformistas.

Al mejorar el pensamiento crítico - reflexivo, como lo indica el Currículo de Educación Obligatoria del (Ministerio de Educación del Ecuador, 2016) las mismas que deben ir acorde a las necesidades del aprendiz en su proceso educativo, fomentando así a corto plazo, realizar un cambio profundo en las instituciones educativas, siendo la lectoescritura el pilar fundamental en todo el proceso de enseñanza-aprendizaje, es así que, el docente impartirá sus clases con estrategias colaborativas de aprendizajes activos, para incrementar la práctica de lectoescritura, brindando la oportunidad al estudiante que interactúen entre sí, generando espacios para el análisis y aporte de soluciones frente a problemas cotidianos, obteniendo un desarrollo integral con lo cual contribuirá a la sociedad. 
Dentro del impacto social que tiene, permite desarrollar capacidades cognitivas en el ámbito investigativo, donde se busca despertar el interés en el estudiante, mediante trabajo colaborativo, haciendo uso apropiado de los elementos, lo que permitirá implementar e innovar la labor docente para un aprendizaje significado.

En la ciudad de Machala donde se desarrollar el actual estudio existe muy poca evidencia de la dificultad que tienen los niños al desarrollar la lectoescritura, más bien las experiencias obtenidas como docente ha permitido detectar ciertas dificultades y errores al momento de aprender a leer y escribir. Según (Puñales Ávila Lucía \& Fundora Martínez , 2017) es fundamental que los docentes tengan un amplio conocimiento sobre estrategias metodológicas activas, para ser incorporadas en el proceso de enseñanza, considerando las características de cada estudiante, seleccionar las más acertadas para alcanzar la construcción de su propio conocimiento.

(Figueroa \& Aillon, 2015) determinaron que un grupo de estudiantes generó liderazgo democrático, al compartir tareas, sus presentaciones grupales, se caracterizaron por un buen nivel de preparación y coordinación que se tradujo en logros propuestos. Esto permitió resaltar lo ventajoso y provechoso que significa el trabajo colaborativo, una vez más queda afianzado este criterio, brindándole al estudiante la oportunidad de participar, adquirir liderazgo entre ellos y lo más sobresaliente la obtención de nuevos conocimientos aplicables a su vida cotidiana.

Es decir (Mora Reyes \& Morales Rivera, 2016) manifiestan sobre la etapa diagnóstica de la lectoescritura, existen ciertas falencias por parte de los estudiantes, puesto que demuestran inseguridad al momento de realizar lectura y descripción de las imágenes. Confirmando el poco interés del estudiante en participar de manera individual, o aislada, más bien es un indicativo para que el docente, incremente metodologías activas como el trabajo colaborativo, permitiendo la integración de los ellos.

En los actuales momentos es prescindible que el docente se encuentre en continua renovación metodológica para mejorar el proceso de enseñanza - aprendizaje es así que (Silva Quiroz, 2010) indica que los estudiantes experimentan cambios tales como, 
luego de ser los receptores de las enseñanzas impartidas por sus docentes para convertirse en los protagonistas de su propio conocimiento.

Por consiguiente existen las diversas metodologías activas como: el aprendizaje colaborativo, estudio de casos, clase invertida, aprendizaje basado en problemas y ( González Fernández \& Carrillo Jácomo, 2016), manifiestan que el aprendizaje colaborativo es una metodología que se fundamenta en la creación de pequeños grupos de estudiantes que colaboran entre sí, para alcanzar objetivos en común, interviene directamente el compañerismo, la ayuda mutua entre los integrantes del equipo, es decir fomenta las relaciones interpersonales.

Resultando ser una renovación pedagógica propia de las comunidades participativas tal como lo indica (Gonzálvez Pérez, García López , \& Traver Marti , 2011) sostienen que el aprendizaje colaborativo permite una mayor participación, interacción, responsabilidad e implicación de parte de los estudiantes para desarrollar habilidades sociales, obteniendo así el mejoramiento de la convivencia social y por ende responsabilidad y liderazgo de cada uno de los integrantes tal como lo sostienen (Borrás Campins \& Sabater Malondra, 2017). El aprendizaje colaborativo según (Herrada Valverde \& Baños Navarro, 2018) es el resultado de la interacción de cada miembro del equipo, compartiendo sus aportaciones, tareas obteniendo un mayor aprendizaje, y el logro de sus propios objetivos.

Según los resultados de (Fuentes Fuentes \& Calderín Careth , 2017) indican que generalmente se debe empezar por ciertos juegos, dinámicas que busquen despertar el interés y curiosidad, para luego abordar textos complejos como son cuentos, fabulas, leyendas, elementos seleccionados de manera informal. La estrategia de iniciar con los conocimientos previos al tema a tratar, resulta atractivo para los niños, ellos logran identificarse con alguna leyenda, cuento o historieta, despertando en ellos la interacción activa lo que permite acoplarse al trabajo colaborativo contribuyendo con ideas claras, acordes al tema lo que les permite cumplir con las tareas encomendadas. 
Es así que se logra entender a través de la nueva escuela, que la enseñanza es aquella que se ubica en el desarrollo de habilidades de los estudiantes a través relacionarse en un grupo de trabajo, permitiéndoles dialogar, debatir, opinar y establecer acuerdos del tema abordado, (Revelo Sánchez \& Collazos Ordoñez, 2018) indican que el aprendizaje colaborativo se fundamenta en el aprendizaje grupal, donde adquiere mucho más conocimiento mucho, que, si lo realizara por sí solo, fruto de la interacción del equipo, llegando a generar un proceso de construcción del conocimiento.

A través de trabajo colaborativo los integrantes del equipo, adquieren mayor conocimiento, porque interactúan entre sí, incrementando de esta manera el cooperativismo entre ellos, permitiéndoles adquirir responsabilidades, compromisos, ayudándose mutuamente, mientras que trabajando de forma aislada poco o nulo sería la adquisición de conocimiento sobre temas tratados. Existen cinco elementos que conforman el trabajo colaborativo como son: cooperación, responsabilidad, comunicación, trabajo en equipo, y autoevaluación. Al hablar sobre trabajo colaborativos referirse a un equipo de trabajo, que comparten sus expectativas, objetivos con respecto a tema, permitiéndoles reflexionar sobre qué tan preparados se encuentran.

\section{Lectoescritura}

Como indica (Franco Montenegro, Cárdenas Rodríguez, \& Santrich Sánchez, 2016) antes de la edad preescolar del niño se origina el acercamiento directo con la lectura, desarrollando en él habilidades que le permiten comprender a través de imágenes con la ayuda de un adulto para su interpretación, involucrándose con la lectura durante toda su etapa de vida de manera informativa, educacional como de entretenimiento. Esto ha permitido que los docentes identifiquen desinterés, ciertas dificultades que presentan los estudiantes en desarrollo de la lectura, reflejándose notablemente en el proceso de enseñanza -aprendizaje. 
(Ballestas Camacho, 2015) sostiene que tanto la lectura como la escritura constituyen los dos grandes pilares de la educación general básica los cuales están inmersos de forma transversal en el proceso educativo, representando tanto un fin como un medio, es decir permitiendo la elaboración del conocimiento, incorporado ciertas estrategias saberes que contribuyen en la formación del niño. Es así como lograron establecer que la lectoescritura afecta, involucra capacidades que intervienen en la percepción, comprensión, creatividad para resolver situaciones, los docentes no favorecen los procesos cognitivos y las habilidades lectoras en los estudiantes. Con esto se consolida el criterio de la interrelación que existe entre la lectoescritura y el desarrollo académico de los niños, complementando directamente con el poco interés del docente en, incorporar u optimizar metodologías activas en su plan de clase, para de esta manera conseguir el aprendizaje significativo (Saltos-Cedeño, et al., 2020).

(Caballeros Ruiz, Sazo, \& Gálvez Sobral, 2015) concuerdan que la capacidad de adquirir más conocimientos radica en la importancia de la lectura es por esta razón que cada vez crece la población estudiantil, esto permite el crecimiento global de los países con la cantidad de personas que no saben leer y escribir. Es así que otras capacidades humanas como hablar, caminar, escuchar, partiendo del hecho que para saber leer necesariamente debe pasar por un proceso de aprendizaje (Vélez-Loor, et al., 2020). Permitiendo alcanzar los mejores resultados, sin dejar las características de cada persona y su respectivo entorno es así que un alto índice de estudiantes que presentan inconvenientes de aprendizaje en lectoescritura.

Mientras tanto los estudios realizados por (González-Valenzuela, Martín Ruiz, \& Delgado Ríos, 2012) indican que la alfabetización diaria y constante desde los primeros niveles de educación, permiten disminuir notablemente la dificultad del aprendizaje de lectoescritura que viene acarreando los estudiantes, mejorando su nivel académico.

La lectura y escritura interactúan desde el inicio preescolar en la vida de los niños, constituyendo un eslabón primordial para su desarrollo en los años venideros, el cual permite al estudiante adquirir una comunicación tanto oral como escrita, es ahí donde el 
docente intervine con un verdadero aprendizaje significativo. (Torres Velásquez, 2016). Entonces se precisa que no existe el verdadero compromiso por parte de los docentes en actualizarse adecuadamente, es decir en dejan su lugar de confort, y ver más allá, que el mismo hecho de incorporar o adicional metodologías activas al plan de clase, los resultados son espectaculares, reflejándose en la participación de los niños, construyendo su conocimiento, lo que les permiten socializarse, estar atentos al desarrollo de todos los integrante del equipo, ayudarse mutuamente, participar y adquirir sus propias experiencias.

A continuación, la presente investigación tiene por objeto analizar el uso del aprendizaje colaborativo para desarrollar la lectoescritura en Educación General Básica.

\section{METODOLOGÍA}

El presente estudio es de carácter descriptivo correlacional no experimental, es decir se limita a observar los acontecimientos sin intervenir, de cohorte transversal (ArgudoTello, Erazo-Álvarez, \& Narváez-Zurita, 2019), porque los datos fueron recolectados en una sola línea de tiempo, con enfoque mixto, en coherencia con los lineamientos de (Hernández Sampier, Fernández Collado, \& Baptista Lucia, 2010) dado que el estudio se caracteriza por la combinación de los enfoques cuantitativo y cualitativo.

La población está constituida por 110 docentes de educación general básica del Circuito 06_07_08_09, perteneciente al Distrito 07D02, del sector público con un muestreo aleatorio simple se calcula la muestra con el $95 \%$ de confiabilidad y con el margen de error del 5\% dando a 67 docentes encuestados, en primer lugar, se procedió a indagar en fuentes bibliográficas a través de google académico, artículos científicos, acerca del conocimiento que tienen los docentes sobre lectoescritura y la incorporación del aprendizaje colaborativo.

Se complementó con la investigación de campo mediante las encuestadas elaboradas a través de un instrumento preciso y conciso con absoluta confiabilidad, cuya finalidad es determinar el inconveniente que se origina en los niños cuando ellos aplican el 
conocimiento adquirido en su diario vivir, obteniendo información significativa (RomoJaramillo, Erazo-Álvarez, Narváez-Zurita, \& Moreno, 2020).

Para efectuar el abordaje cuantitativo se recurrió a la encuesta elaborada en Google Drive acorde a la escala de Likert, conformada por 17 preguntas validada mediante el modelo de Alfa de Cronbach cuyo valor es de 0,76 con la finalidad de contextualizar el entorno de esta investigación, tal como lo indica (García Alcaraz, Alfaro Espín, Hernández Martínez, \& Molina Alarcón, 2006), sobre la información recopilada por medio de parámetros que determinan las variables implicadas en el objetivo de estudio. El análisis fue validado por el programa estadístico software SPSS que mostró en forma descriptiva gráficos estadísticos, tabulación de datos, determinación de variables reflejadas mediante la aplicación de la Chi-cuadrado, que verificó la dependencia entre ellas (Erazo \& Narváez, 2020).

\section{RESULTADOS}

Los resultados obtenidos de las encuestas realizadas a los 70 docentes demostraron claramente que el problema radicó durante todo el proceso de enseñanza-aprendizaje de lectoescritura en los primeros años de estudio de los niños, por la sencilla razón de que no aplicaron ningún tipo de estrategias pedagógicas, ni metodologías activas de ese entonces. Además de esto se utilizó la Estadística Descriptiva para identificar el análisis cuantitativo, lo que permitió el cruce de variables paramétricas y no paramétricas cabe indicar que para la asociación de variables se utilizó el chipcuadrado.

Con tres grupos como son: el aprendizaje colaborativo en clases de lectoescritura/organiza su intervención para el aprendizaje de lectoescritura, listado de metodologías activas que aplica en sus clases/destreza con criterio de desempeño en lectoescritura y por último incorpora aprendizaje colaborativo en clases de lectoescritura/organiza su intervención para el aprendizaje de lectoescritura. 
Por lo tanto es fundamental resaltar los datos obtenidos a través de la indagación sobre de una de las variables de mayor enfoque para este trabajo investigativo, como es el porcentaje de los docentes donde consideraron que el problema de lectoescritura se origina en los primeros niveles de educación, lo que se refleja en la gráfica estadística a continuación.

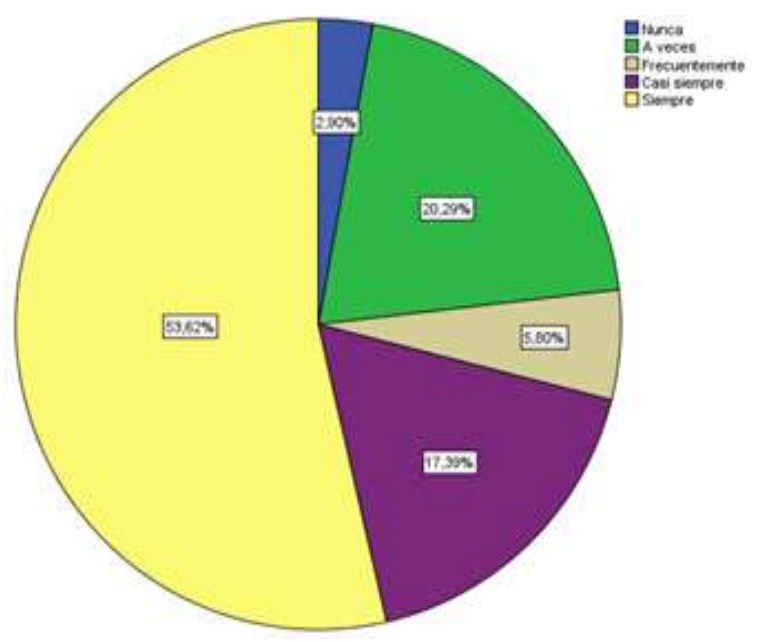

Figura 1. Docentes que coinciden que la lectoescritura es un problema. Fuente: Elaboración propia

Es así que la gráfica indica que el $53,62 \%$ de docentes encuestados siempre concuerdan que lectoescritura constituye un verdadero problema para el aprendiza de los niños, debido que tanto profesores como familiares intervienen directamente en el desarrollo del conocimiento, siendo estos los guías para lograr lo establecido, mientras que un $20,29 \%$ señaló que a veces ese problema vienen acarreando desde la preparatoria, por otro lado el $17,39 \%$ señalan que casi siempre el problema de lectoescritura radica en los primeros años de estudio, el 5,80\% se mantienen que frecuentemente se da ese inconveniente y apenas el 2,90\% corresponde a los docentes que nunca se percataron de dicha falencia ubicarla en los primeros años del estudiante, a pesar que es un valor mínimo, lo que conlleva pensar que existe docentes que no vinculan directamente con el aprendiza- significativo. 
Con el afán de obtener mayor información que aporte a la presente investigación se plantearon además ciertas hipótesis de relación entre diferentes variables, para luego ser analizadas a través de la utilización del software bajo la prueba de Chi-cuadrado para el contracte de las mismas, lo que permitió encontrar datos muy significativos que se encuentran en las diferentes tablas a continuación.

\section{Tabla 1}

Aprendizaje Colaborativo en clases de lectoescritura y organiza intervención para el

\begin{tabular}{|c|c|c|c|c|c|c|}
\hline & \multicolumn{4}{|c|}{$\begin{array}{l}\text { Organiza intervención para el aprendizaje de } \\
\text { lectoescritura }\end{array}$} & \multirow{2}{*}{ Total } \\
\hline & & A veces & Frecuentemente & $\begin{array}{c}\text { Casi } \\
\text { siempre }\end{array}$ & Siempre & \\
\hline Aprendizaje & Frecuentemente & 2 & 1 & 1 & 7 & 11 \\
\hline Colaborativo & Casi siempre & 1 & 1 & 1 & 9 & 12 \\
\hline $\begin{array}{l}\text { en clases de } \\
\text { lectoescritura }\end{array}$ & Siempre & 0 & 2 & 0 & 42 & 44 \\
\hline $\begin{array}{l}\text { Total } \\
\text { aprendizaje c }\end{array}$ & le lectoescritura & 3 & 4 & 2 & 58 & 67 \\
\hline \multicolumn{7}{|c|}{ Pruebas de chi-cuadrado } \\
\hline & & \multicolumn{2}{|l|}{ Valor } & \multicolumn{3}{|c|}{ Sig. asintótica (2caras) } \\
\hline \multicolumn{2}{|c|}{ Chi-cuadrado de Pearson } & $12,516^{a}$ & 6 & \multicolumn{3}{|c|}{0,051} \\
\hline \multicolumn{2}{|c|}{ Razón de verosimilitud } & 12,865 & 6 & \multicolumn{3}{|c|}{0,045} \\
\hline \multicolumn{2}{|c|}{ Asociación lineal por lineal } & 8,865 & \multirow[t]{2}{*}{1} & \multicolumn{3}{|c|}{0,003} \\
\hline \multicolumn{2}{|c|}{$\mathrm{N}$ de casos válidos } & 67 & & & & \\
\hline
\end{tabular}

Fuente: Elaboración propia

En la tabla 1 se observó que el nivel de significación es Ho, lo que demostró que estas dos variables no guardan relación entre sí, es decir son no asociadas, tanto el aprendizaje colaborativo en las clases de lectoescritura y la forma como organiza la intervención el docente en el aprendizaje de la misma, este análisis determinó que el $42 \%$ de los docentes siempre incorporaron e I aprendizaje colaborativo dentro de las 
clases de lectoescritura, organizaron su intervención con antelación, mientras que por otro lado el $9 \%$ corresponde al casi siempre los docentes incorporaron el aprendizaje colaborativo por último el 7\%, corresponde a la escala de frecuentemente impartieron sus clases incluyendo el aprendizaje colaborativo y organizando su intervención.

\section{Tabla 2}

Listado de metodologías activas aplica en sus clases y destreza con criterio de desempeño en lectoescritura

\begin{tabular}{|c|c|c|c|c|c|}
\hline & $\begin{array}{r}\text { Dest } \\
\text { A veces }\end{array}$ & $\begin{array}{r}\text { reza con criterio } \\
\text { lectoesc } \\
\text { Frecuentemente }\end{array}$ & $\begin{array}{l}\text { de desempei } \\
\text { ritura } \\
\text { Casi siempre }\end{array}$ & $\begin{array}{l}\text { ר̃o en } \\
\text { Siempre }\end{array}$ & Total \\
\hline \multirow{4}{*}{$\begin{array}{lr}\text { Listado } & \text { de } \\
\text { metodologías } & \text { activas } \\
\text { aplica en sus clases }\end{array}$} & 0 & 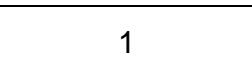 & 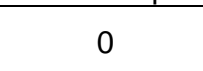 & 1 & 2 \\
\hline & 0 & 0 & 1 & 0 & 1 \\
\hline & 1 & 2 & 7 & 51 & 61 \\
\hline & 0 & 1 & 1 & 1 & 3 \\
\hline $\begin{array}{c}\text { Aprendizaje Basado en } \\
\text { Problemas }\end{array}$ & 0 & 0 & 0 & 2 & 2 \\
\hline tivnetilias & 1 & 4 & 9 & 55 & 69 \\
\hline \multicolumn{6}{|l|}{ Prueba de chi-cuadrado } \\
\hline & Valor & gl & \multicolumn{3}{|c|}{ Sig. asintótica (2caras) } \\
\hline Chi-cuadrado de Pearson & $21,066^{a}$ & 12 & \multicolumn{3}{|c|}{0,049} \\
\hline Razón de verosimilitud & 13,030 & 12 & \multicolumn{3}{|c|}{0,367} \\
\hline Asociación lineal por lineal & 0,805 & 1 & \multicolumn{3}{|c|}{0,370} \\
\hline $\mathrm{N}$ de casos válidos & 69 & & & & \\
\hline
\end{tabular}

Fuente: Elaboración propia

Se determinó en la tabla 2 que el nivel de significación es $\mathrm{H}_{1}$ lo que demostró que estas dos variable son asociadas entre sí, al aplicar metodologías activas permitió trabajar con mayor exactitud las destrezas con criterio desempeño, es así el 51\%, de los docentes utilizaron el aprendizaje colaborativo, desarrollaron siempre las destreza con criterio de desempeño, un $2 \%$ trabajó con aprendizaje basado en problemas, mientras que el $1 \%$ correspondió tanto al aprendizaje basado en proyectos, y a la gamificación. 
Esto permitió determinar que los docentes conocen sobre metodologías activas, pero que lastimosamente no aplican de manera adecuada en sus diarias enseñanzas, a pesar que trabajan con destrezas con criterio de desempeño otorgadas por el currículo nacional de educación.

\section{Tabla 3}

Destreza con criterio de desempeño en lectoescritura * Avance en aprovechamiento de los estudiantes en lectoescritura

Avance en aprovechamiento de los estudiantes en lectoescritura

\begin{tabular}{llccccc} 
& & A veces & Frecuentemente & Casi siempre & Siempre & Total \\
\hline Destreza con criterio de & A veces & 1 & 0 & 0 & 0 & 1 \\
desempeño & en Frecuentemente & 1 & 1 & 1 & 1 & 4 \\
lectoescritura & Casi siempre & 0 & 2 & 5 & 2 & 9 \\
& Siempre & 0 & 3 & 16 & 37 & 56 \\
Total & & 2 & 6 & 22 & 40 & 70
\end{tabular}

Pruebas de chi-cuadrado

\begin{tabular}{cccc} 
& Valor & gl & Sig. asintótica (2caras) \\
Chi-cuadrado de Pearson & $51,903^{\mathrm{a}}$ & 9 & 0,000 \\
Razón de verosimilitud & 22,082 & 9 & 0,009 \\
Asociación lineal por lineal & 20,449 & 1 & 0,000 \\
N de casos válidos & 70 & & \\
\hline
\end{tabular}

Fuente: Elaboración propia

Los resultados de la tabla 3, entre el cruce de dos variables indicaron que existe una estrecha relación entre las mismas, tales como la destreza con criterio de desempeño en lectoescritura y avance en el aprovechamiento de los estudiantes, es decir que el nivel de significación es de $\mathrm{H}_{1}$ lo que corresponde al $37 \%$ los docentes demostraron que siempre utilizaron destrezas con criterio de desempeño, con esto permitió desarrollar el avance diario de las asignaturas, mientras que el $2 \%$ casi siempre, aplicaron dichas destrezas quedando el $1 \%$ que frecuentemente consideró ciertas destrezas para 
obtener el avance académico de los estudiantes.

Con los resultados obtenidos en la presente investigación demuestran que existen ciertas falencias que presentan los estudiantes a cerca de lectoescritura en los primeros años de estudio, las cuales se agudizan por la escasa incorporación de metodologías activas como el aprendizaje colaborativo, es así que paralelamente, el estudio realizado por (Ballestas Camacho, 2015) concuerda e indica que la utilización de medios didáctico exclusivo para la enseñanza de lectoescritura en los primeros niveles de estudio de los niños, constituye en uno de los factores por los cuales los estudiantes presentan algún tipo de inconformidad al momento de realizar sus tareas, al no contar con el apoyo pedagógico que ameritan desde el punto de vista de metodologías acordes para la enseñanza de lectoescritura.

Mientras tanto (Mora Reyes \& Morales Rivera, 2016) manifiestan que su investigación está concentrada en nuevas estrategias educativas para contribuir en los procesos de aprendizajes de los niños en los primeros años de estudio, observándose ciertas dificultades en lectura y escritura debido a la escasa incorporación de un aprendizaje significativo, a través del trabajo colaborativo, y mínimo acompañamiento de los padres direccionan al fracaso estudiantil.

Coincidiendo con (Franco Montenegro, Cárdenas Rodríguez, \& Santrich Sánchez, 2016) de que el ser humano tiene acercamiento con la lectura desde su edad preescolar, siendo así, la lectoescritura estará presente en todas las etapas de la vida, esto permite que los docentes identifiquen en los estudiantes dificultades al momento de leer el, enunciado o el párrafo de alguna historieta, es decir cuando aplica la lectoescritura. 


\section{PROPUESTA}

A través de la historia la lectura ha sido un instrumento indispensable para el desarrollo de la humanidad, por ende la comprensión lectora va de la mano con ella partiendo del hecho que la lectoescritura constituye una piedra angular dentro del sistema educativo, es ahí donde radica la importancia de llegar con un aprendizaje significativo por parte del docente, a los niños desde su edad preescolar, otorgando estrategias activas como el aprendizaje colaborativo, permitiendo al docente dejar de ser el emisor del conocimiento para adquirir su nuevo rol como un mediador del aprendizaje, otorgando las estrategias activas específicamente el aprendizaje colaborativo brindándole la oportunidad al estudiante en convertir en protagonista de su propio conocimiento.

Es ahí donde va enfocada la propuesta, que consiste en mejorar el aprendizaje de la lectoescritura en niños de educación general básica optimizando la utilización de metodologías activas como es el aprendizaje colaborativo, con la finalidad de obtener resultados acordes a los niveles de enseñanza educativa, promoviendo actividades de participación, integración y trabajo en equipos.

La optimización del aprendizaje colaborativo como estrategia de las metodologías activas para mejorar el aprendizaje de la lectoescritura en educación general básica está constituido por tres fases que se explican a continuación: 

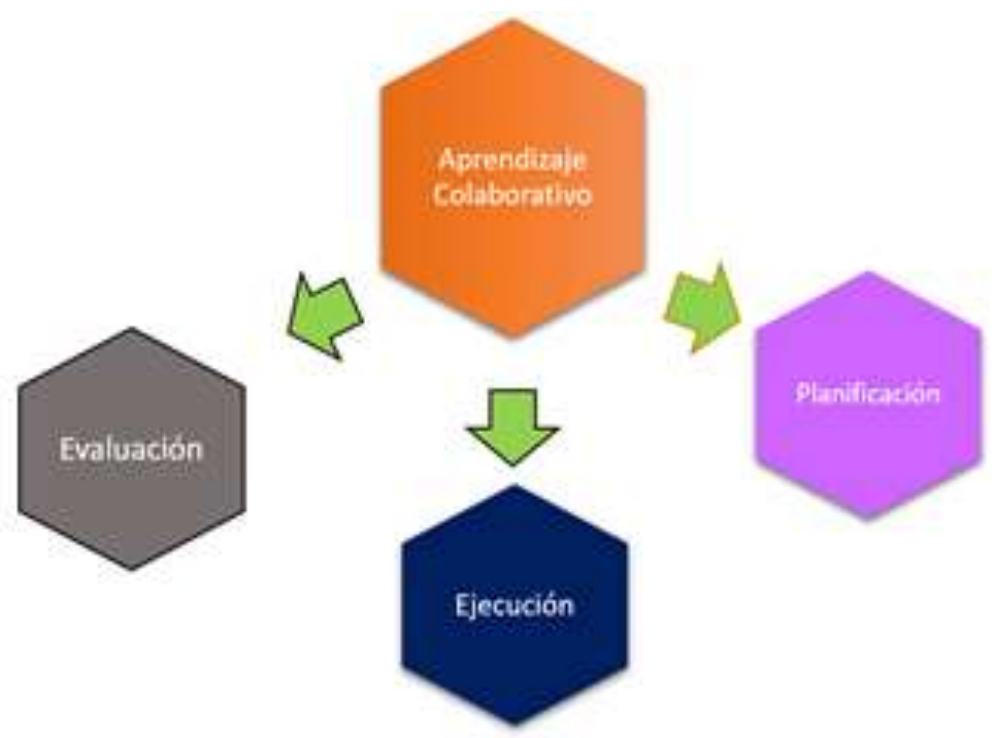

Figura 2. Estructura del Aprendizaje Colaborativo. Fuente: Elaboración propia.

Se establece implementar el aprendizaje colaborativo como estrategia para mejorar el aprendizaje de lectoescritura mediante un proceso que consta de tres componentes como son la planificación, ejecución y evaluación.

Planificación.- Considerando que para realizar la propuesta se partió de la introducción donde se enmarca la importancia de utilizar aprendizaje colaborativo en el desarrollo de lectoescritura, obteniendo resultados estadísticos favorables donde la mitad de los docentes encuestados incorporan ciertas metodologías activas en sus clases, en primera instancia esto permite dialogar con los docentes a cerca de las experiencias adquiridas, luego de esto se realizará la capacitación sobre el uso adecuado del aprendizaje colaborativo en el desarrollo de lectoescritura en educación general básica, seleccionando los objetivos para la incorporación de esta metodología activa conjuntamente con las destrezas con criterio de desempeño que constan en el currículo de educación, como última parte de esta planificación se plantearán tanto las metas que se obtendrán al aplicar este tipo de aprendizaje, como los resultados que se esperan alcanzar. 
Ejecución.- A continuación esta fase de ejecución es la más relevante, porque permite llevar a cabo lo estipulado anteriormente en la planificación, es aquí donde el docente imparte sus clases incorporando adecuadamente el aprendizaje colaborativo, animando día a día a sus estudiantes para que participen, integren de forma activa a esta nueva metodología, permitiéndoles a ellos ser los constructores de su propio conocimientos, por su parte los educadores realizarán observaciones a los diferentes grupos resaltando siempre la integración, relación mutua, el compañerismo y el aprendizaje significativo de ellos.

Evaluación.- Como última fase de esta propuesta está la evaluación que consiste en el análisis del desarrollo y cumplimiento de las actividades acordadas en las dos fases anteriores de la propuesta como es la planificación y ejecución, como parte complementaria los docentes intervienen en un diálogo para dar a conocer los resultados que obtuvieron luego de las observaciones directas en sus horas clase a los estudiantes, además esta fase permite conocer si se logró alcanzar tanto el objetivo plateando cono la meta trazada.

Se elaborará un cuestionario en google drive, que se enviará por WhatsApp, dirigido para los docentes así también es conocer desde el punto de vista de los estudiantes, que permitirá indagar, obtener los resultados de forma cualitativa.

\section{CONCLUSIONES}

La educación constituye un pilar fundamental para el desarrollo de la sociedad, encontrándose inmersa desde los primeros niveles de pre escolaridad de la humanidad, es un proceso que exige un cambio continuo, actualmente el docente debe busca las estrategias adecuadas y específicas para llegar al aprendizaje significativo, aplicando metodologías activas, las mismas que promueven el desenvolvimiento de los estudiantes lo que conlleva a desarrollar actividades nuevas de interés general, creando un ambiente acorde, estable, participativo, de mutua colaboración entre los integrante del equipo, brindando así la oportunidad al niño, joven de ser el constructor de su 
propio conocimiento.

Dado que la lectura y escritura interactúan gradualmente en el proceso de enseñanza aprendizaje, por lo tanto, la lectoescritura es el reconocimiento de letras, símbolos, es decir el producto final de todo lo que se imparte a los estudiantes durante todo el proceso educativo.

La lectoescritura es parte de la asignatura de Lengua y Literatura, razón por la cual el docente de educación general básica debe brindar todas las pautas necesarias y estar comprometido con el desarrollo académico de sus estudiantes, partiendo de que esta asignatura empieza desde los primeros niveles de pre escolar reflejándose en todo su periodo estudiantil.

A través de la utilización de la encuesta, con diseño no experimental de corte transversal afianzando los resultados, es así que los niveles de confiabilidad permitieron demostrar estadísticamente la relación existente entre el aprendizaje colaborativo y lectoescritura, la participación docente es importante brindando la oportunidad de mejorar el proceso de enseñanza-aprendizaje, la propuesta planteada resalta la incorporación de metodologías activas, a través del aprendizaje colaborativo.

\section{FINANCIAMIENTO}

No monetario

\section{AGRADECIMIENTOS}

A los docentes de educación general básica del Circuito 06_07_08_09, perteneciente al Distrito 07D02, del sector público. 


\section{REFERENCIAS}

Argudo-Tello, K. J., Erazo-Álvarez, J. C., \& Narváez-Zurita, C. I. (2019). Evaluación de Control Interno en Riesgos Estratégicos para la Dirección de Planificación de la Universidad de Cuenca. [Evaluation of Internal Control in Strategic Risks for the Planning Direction of the University of Cuenca]. Revista Arbitrada Interdisciplinaria Koinonía, 67-96. doi:http://dx.doi.org/10.35381/r.k.v4i1.372

Ballestas Camacho, R. (2015). Relación entre TIC y la adquisición de habilidades de lectoescritura en alumnos de primer grado de básica primaria [Relationship between ICT and the acquisition of literacy skills in first grade primary school students]. Investigación \& Desarrollo, 338-368. Obtenido de https://url2.cl/pk3lk

Borrás Campins , M., \& Sabater Malondra , M. (2017). Cómo puede el aprendizaje colaborativo modificar la organización en la escuela[How collaborative learning can change organization in school ]. Aula de innovación educativa, 260, 55-59. Obtenido de https://url2.cl/ELV1z

Caballeros Ruiz, M., Sazo, E., \& Gálvez Sobral, J. (2015). El aprendizaje de la lectura y escritura en los primeros años de escolaridad: experiencias exitosas de Guatemala[Learning to read and write in the first years of schooling: successful experiences in Guatemala]. Interamerican Journal of Psychology, 48(2), 212-222. Obtenido de https://url2.cl/JbVpM

Cano de la Cruz, Y., Aguiar Monar, J., \& Mendoza Román, M. (2017). Metodologías activas:una necesidad en la unidad educativa reino de inglaterra[Active methodologies: a necessity in the Kingdom of England educational unit]. Revista Educación, 43(2), 1-10. Obtenido de https://url2.cl/bgEv4

Erazo, J. C., \& Narváez, C. I. (2020). La gestión del capital intelectual y su impacto en la efectividad organizacional de la industria de cuero y calzado en la Provincia de Tungurahua - Ecuador [The management of intellectual capital and its impact on the ] Recuperado de: https://n9.cl/52li. Revista Espacios, 254-271.

Figueroa, B., \& Aillon, M. (2015). Escritura académica de un ensayo mediado por el aprendizaje colaborativo virtual [Academic writing of an essay mediated by virtual collaborative learning]. Estudios mpedagógicos, 79-99. Obtenido de https://url2.cl/m9Nd1 
Franco Montenegro, M., Cárdenas Rodríguez, R., \& Santrich Sánchez, E. (2016). Factores asociados a la comprensión lectora en estudiante de noveno grado de Barranquilla [Factors associated with reading comprehension in a ninth grade student of Barranquilla]. Psicogenrte, 19(36), 296-310. Obtenido de https://url2.cl/aplTT

Fuentes Fuentes, L., \& Calderín Careth , N. (2017). Prácticas en la enseñanza de la lectura en grado primero en instituciones oficiales municipales [Practices in the teaching of reading in first grade in official municipal institutions]. Opción, 33(82), 488-515. Obtenido de https://url2.cl/XaTqw

García Alcaraz, F., Alfaro Espín, A., Hernández Martínez, A., \& Molina Alarcón, M. (2006). Diseño de Cuestionarios para la recogida de información: metodología y limitaciones[Design of questionnaires to collect information:methodology and limitation]. Revista clínica de medicina y familia., 1(5), 232-236. Obtenido de https://url2.cl/bBTp3

González-Valenzuela, M., Martín Ruiz, I., \& Delgado Ríos, M. (2012). Teaching Literacy and Decreased[Enseñanza de alfabetización y disminución]. Psicodidáctica, 17(2), 253-269. Obtenido de https://url2.cl/pC878

Gonzálvez Pérez , V., García López , R., \& Traver Marti , J. (2011). El aprendizaje cooperativo desde una perspectiva ética[Cooperative learning from an ethical perspective]. Estudios sobre educación, 21, 181-197. Obtenido de https://url2.cl/nf5ft

González Fernández , N., \& Carrillo Jácomo, G. (2016). El aprendizaje cooperativo y la flipped classroom: una pareja ideal [Cooperative learning and the Flipped Classroom: an ideal couple]. Aularia, 5(2), 43-48. Obtenido de https://url2.cl/AVDe3

Hernández Sampier, R., Fernández Collado, C., \& Baptista Lucia, M. (2010). Metodología de investigación [Research methodology]. Obtenido de https://url2.cl/V8pr9

Herrada Valverde , R., \& Baños Navarro , R. (2018). Aprendizaje cooperativo a través de las nuevas tecnologías: Una revisión @tic [Cooperative learning through new technologies: an @tic review ]. Innovación educativa, 18-26. Obtenido de https://url2.cl/W9mZx

Ministerio de Educación del Ecuador. (2016). MINEDUC. Obtenido de https://educacion.gob.ec/ 
Mora Reyes , J., \& Morales Rivera, S. (2016). Fortalecimiento en los procesos lectoescritos en primera infancia a través de blended-learning [Strengthening of reading and writing processes in early childhood through blended-learnig]. Iberoamericana, 14(1), 117-135. Obtenido de https://url2.cl/WLNEF

Puñales Ávila Lucía , L., \& Fundora Martínez , C. (2017). La enseñanza de la lectoescritura en la Educación Primaria: reflexión desdelas dificultades de aprendizaje[The teaching of literacy in Primary Education: reflection from learning difficulties]. Atenas, 1(37), 125-138. Obtenido de https://url2.cl/F6lxT

Romo-Jaramillo, M. V., Erazo-Álvarez, J. C., Narváez-Zurita, C. I., \& Moreno, V. P. (2020). Estrategias de redes sociales para la promoción de macanas artesanales de la provincia del Azuay [Social media strategies for the promotion of artisan macanas in the province of Azuay]. Revista Arbitrada Interdisciplinaria Koinonía, 545-575. doi:http://dx.doi.org/10.35381/r.k.v5i10.705

Revelo Sánchez, O., \& Collazos Ordoñez, C. (2018). El trabajo colaborativo como estrategia didáctica para la enseñanza y aprendizaje de la programación: una revisión sistemática de literatura[Collaborative work as a didactic strategy for programming teaching and learning: a systematic literature review]. Tecnológicas, 1(82), 114-.134. Obtenido de https://url2.cl/mF3rl

Saltos-Cedeño, A., Vallejo-Valdivieso, P., \& Moya-Martínez, M. (2020). Innovación en educación matemática de básica superior durante el confinamiento por COVID19. [Innovation in higher basic mathematics education during confinement by COVID-19]. KPISTEME KOINONIA, $\quad 3(5), \quad 142-161$. http://dx.doi.org/10.35381/e.k.v3i5.723

Silva Quiroz, J. (2010). Un modelo pedagógico virtual en las E-actividades [A virtual pedagogical model in E-activities]. Revista de Educación a distancia, 10(52), 1323. Obtenido de https://url2.cl/Tzb6D

Torres Velásquez, M. (2016). Literacy: Literacy Events in Preschool[Lectoescritura: eventos de literacidad en preescolar]. Iberoamericana para la Investigación y el Desarrollo Educativo, 6(12). Obtenido de https://url2.cl/bz1Vx

Vélez-Loor, M., Vallejo-Valdivieso, P., \& Moya-Martínez, M. (2020). Recursos didácticos virtuales en proyectos de ciencias naturales en período de confinamiento por COVID-19. [Virtual teaching resources in natural science projects in confinement period by COVID-19]. EPISTEME KOINONIA, 3(5), 183-201. http://dx.doi.org/10.35381/e.k.v3i5.760 
EPISTEME KOINONIA

Revista Electrónica de Ciencias de la Educación, Humanidades, Artes y Bellas Artes

Año III. Vol III. N6. Julio - Diciembre 2020

Hecho el depósito de Ley: FA2018000022

ISSN: $2665-0282$

FUNDACIÓN KOINONIA (F.K)

Santa Ana de Coro, Venezuela

Luz María Arias-Jaramillo; Darwin Gabriel García-Herrera; Ana Zulema Castro-Salazar; Juan Carlos Erazo-Álvarez

(O2020 por el autor. Este artículo es de acceso abierto y distribuido según los términos y condiciones de la licencia Creative Commons Atribución-NoComercial-Compartirlgual 4.0 Internacional (CC BY-NC-SA 4.0)

(https://creativecommons.org/licenses/by-nc-sa/4.0/). 